\title{
Extinction cascades and the distribution of species interactions
}

\author{
Mike S. Fowler \\ M. S. Fowler(mfowler@imedea.uib-csic.es), Integrative Ecology Unit, Dept of Biological and Environmental Sciences, PO Box 65, Viikinkaari 1, \\ FIN-00014 Helsinki University, Finland. Present address: Population Ecology Group, IMEDEA (UiB-CSIC), C/ Miquel Marquès 21, 07190 \\ Esporles, Spain.
}

\begin{abstract}
The distribution of interaction strengths among community members has important consequences for assembly processes and community responses to perturbations. Species deletion from communities can trigger cascading extinction events, with strong evidence from empirical and theoretical work. I examined model competitive communities, sequentially assembled using species drawn from a global pool with interaction strengths described by different distribution shapes (uniform or beta), with the same mean and variance. As community size increased, it became harder to assemble communities drawn from a uniform distribution compared to a beta distribution. The distribution of interaction values in the assembled communities differed from the shape of the initial distribution. The distribution shape and the relative abundance of the deleted species also had strong impacts on the probability of extinction cascades following primary species removal. Extinction cascades occurred in communities with a higher mean and variance of interaction strengths before the primary extinction. Those species lost had negative equilibrium densities and tended to be the least abundant, when assessed following the reorganisation that occurred after the primary and subsequent extinctions. Knowledge of the shape of the distribution of interaction strengths from real communities will allow us to make better predictions about which species are most at risk in extinction cascades under natural circumstances.
\end{abstract}

The loss of species from ecological communities and the effects this has on the remaining community is a major topic of current interest. In many cases, the loss of a single species leads to further community members declining in abundance, potentially to extinction, termed 'cascading extinction events'. Extinction cascades have been noted in field (Paine 1966, Berlow 1999, Paine and Trimble 2004) and micro-/ mesocosm studies (Fox and McGrady-Steed 2002, Bruno and O'Connor 2005) and are predicted from theoretical work on competitive communities (Lundberg et al. 2000, Fowler and Lindström 2002, Fowler 2005) and trophically structured food-webs (Pimm 1980, Borrvall et al. 2000, 2006, Dunne et al. 2002, Allesina and Bodini 2004, Petchey et al. 2004, Quince et al. 2005, Allesina et al. 2006, Eklöf and Ebenman 2006, Jonsson et al. 2006, Petchey et al. 2008), with extinction cascades in turn impacting upon ecosystem functioning (Petchey et al. 2004, Thébault et al. 2007).

The fact that extinction cascades occur in purely competitive communities demonstrates that factors other than resource depletion/removal can be a route to further species loss. Indirect effects in purely antagonistic networks facilitate persistence in competitive communities whose species do not coexist in other combinations. Understanding why extinction cascades occur following the removal of species and predicting which species will be involved remain important challenges for contemporary ecological theory. Investigations within a single trophic level are required (Berlow et al.
2004) - they give insight into alternative explanations for extinctions and can provide useful information that can be extended and applied to more complex trophic models.

The mean and variance of interaction strengths, and the balance of weak and or strong interactions can affect various community processes (reviewed by McCann 2000, Berlow et al. 2004, see also Fowler et al. 2002, Jonsson et al. 2006, Fowler 2009), as can the distribution shape (Emmerson and Yearsley 2004). Here I explicitly compare different distributions of interaction strengths with the same expected value (mean) and variance, to examine how distribution shape may influence the assembly of competitive communities and their responses to species loss.

Estimating the strength of species interactions is notoriously difficult under natural circumstances (Abrams 2001, Berlow et al. 2004), although methods have been developed to attempt this (Ives et al. 2003). For this reason, it is helpful to use some simple proxy that summarises the effects of all species interactions, and see if useful predictions on community processes can be made based on this. Using the relative abundance of species within model competitive communities has been shown to be a useful proxy when predicting extinctions in communities under different disturbance scenarios (Fowler 2005, Enberg et al. 2006, Ruokolainen et al. 2007). Here I utilised the relative abundance of species within communities to assess how the remaining community responds to the deletion of the least or most abundant species compared 
to random species removal and characterise which species are involved in extinction events. This also relates to recent work on network theory that shows that selective removal of species from a food-web leads to different patterns of cascading extinctions compared to random removal (Ives and Cardinale 2004, Montoya et al. 2006).

In this work I investigated the following main questions: (1) how do different distribution patterns of interaction strengths (uniform or beta) affect the assembly of competitive communities, when the mean and variance of the global distributions are equal? (2) Do communities assembled under these different conditions show different responses to the deletion of a species (primary extinction)? (3) Who is expected to be involved in cascading extinctions when different community members are deleted? I examined these questions in communities formed with sequential assembly rules (main text) and in randomly assembled communities (Supplementary material Appendix 1). There are important differences in assembly processes and extinction cascades following primary extinction in communities that differ in their global distribution of interaction strengths. Communities drawn from a uniform distribution were harder to assemble, had a higher probability of extinction cascades and lost more species in those cascades. Communities that suffered extinction cascades had a higher mean and variance in interaction strength than those that do not. This study represent the first investigation into the effect of the distribution shape of interaction strengths on community assembly and cascading extinction processes.

\section{Methods}

I modelled multi-species population growth using a discretetime logistic (Ricker) type model with Lotka-Volterra competitive interactions between species pairs. While this model only considers a single trophic level, trophically structured models often rely on the same basic assumptions for the basal trophic layer (Emmerson et al. 2004, Thébault et al. 2007, Petchey et al. 2008, Discussion), therefore this model considers effects occurring within a trophic level or guild. The choice of this model does not affect any of the underlying methods detailed below, and in this case the discrete model relies upon exactly the same functional forms as the continuous time logistic function. The density $\mathrm{N}$ of species $\mathrm{i}$ is calculated over successive generations $(t)$ as follows

$$
N_{i, t+1}=N_{i, t} \exp \left(r_{i}\left[1-\frac{\sum_{j=1}^{S} \alpha_{i j} N_{j, t}}{K_{i}}\right]\right)
$$

in an $S$ species community where $r_{i}$ and $K_{i}$ are the species specific intrinsic growth rates and carrying capacities respectively. The between species competition coefficient $\alpha_{i j}$ represents the per-capita effect of species $j$ on the per-capita growth rate of species $i$. The carrying capacities of all species in the community can be represented as a vector $\mathbf{K}$, which along with the matrix of competition coefficients $\mathbf{A}$ can be used to calculate the vector of equilibrium densities $\mathbf{N}^{*}$ (May 1973)

$$
\mathbf{N}^{*}=\mathbf{A}^{-1} \mathbf{K}
$$

If all elements of $\mathbf{N}^{*}$ are greater than zero, the community is said to be feasible. In the following analyses, all values of $\mathrm{r}_{\mathrm{i}}$ and $\mathrm{K}_{\mathrm{i}}$ were set to 1.75 and 1 respectively for simplicity and in order to generate comparable results to earlier work. All intraspecific competition coefficients $\left(\alpha_{\mathrm{ii}}\right)$ were set equal to one, with off diagonal elements drawn at random from a given distribution as outlined below.

The dominant eigenvalue $\left(\lambda_{1}\right)$ of the Jacobian matrix $(\mathbf{J})$ evaluated around the equilibrium of the system of linked equations that govern community population dynamics (Eq. 1) tells us about the local stability of a given community. Each element (row i, column $\mathbf{j}$ ) in $\mathbf{J}$ tells us the effect of a small change in density of species $j$ on the population growth rate of species $i$. The Jacobian matrix for the above system (Eq. 1) is

$$
\mathbf{J}=\left[\begin{array}{cccc}
1-\frac{\mathrm{r}_{1}}{\mathrm{~K}_{1}} \mathrm{~N}_{1}^{*} & -\frac{\alpha_{12} \mathrm{r}_{1}}{\mathrm{~K}_{1}} \mathrm{~N}_{1}^{*} & \cdots & -\frac{\alpha_{1 S} \mathrm{r}_{1}}{\mathrm{~K}_{1}} \mathrm{~N}_{1}^{*} \\
-\frac{\alpha_{21} \mathrm{r}_{2}}{\mathrm{~K}_{2}} \mathrm{~N}_{2}^{*} & 1-\frac{\mathrm{r}_{2}}{\mathrm{~K}_{2}} \mathrm{~N}_{2}^{*} & \cdots & -\frac{\alpha_{2 S} \mathrm{r}_{S}}{\mathrm{~K}_{S}} \mathrm{~N}_{2}^{*} \\
\vdots & \vdots & \ddots & \mathrm{M} \\
-\frac{\alpha_{S 1} \mathrm{r}_{S}}{\mathrm{~K}_{S}} \mathrm{~N}_{S}^{*} & -\frac{\alpha_{S 2} \mathrm{r}_{S}}{\mathrm{~K}_{S}} \mathrm{~N}_{S}^{*} & \cdots & 1-\frac{\mathrm{r}_{S}}{\mathrm{~K}_{S}} \mathrm{~N}_{S}^{*}
\end{array}\right],
$$

which means that while information about species intrinsic growth rates is not required to find their equilibrium densities (Eq. 2), it does affect local stability. If the absolute value of the dominant eigen value of $\mathbf{J}\left(\left|\lambda_{1}\right|\right)$ is less than 1 , the system is said to be locally stable, and any small perturbation in $\mathrm{N}$ will result in the system returning to its equilibrium. If $\left|\lambda_{1}\right|>1$, the equilibrium is locally unstable, and any small perturbation will grow over time until the system settles at a new state. In the case where $r>2$, this can result in regular population cycles or more complex dynamics. However, if $\mathrm{r}<2$, as in this particular system, perturbations away from a locally unstable equilibrium will lead to extinction of one or more species, as the new equilibrium state the system settles at corresponds to the extinction boundary $\left(\mathrm{N}_{\mathrm{i}}=0\right)$ of those species that become extinct. Here I focus on the case of $r_{i}<2$ to allow easy comparison with previous works, and simplify the amount of work required to do to establish which species are involved in cascading extinction events. This assumption also means that results presented here do not change if the model is studied in continuous time, with the relevant local stability conditions being that the real parts of all $\lambda$ 's must be less than 0 . Another useful feature is that global stability conditions are the same as locally stability conditions in this model (Turrelli 1978), so any feasible and locally stable community will return to the internal equilibrium from anywhere in the positive density phase-space.

\section{Global distribution of interaction strengths}

I assumed that the component members of any community were drawn from a global species pool, where the potential 
interspecific interaction strengths could be described by a specific distribution form, with associated mean and variance. Here, I employed either a beta or a uniform distribution, keeping a constant expected value $\left(\mu=1 /{ }_{4}\right)$, and variance $\left(\sigma^{2}=1 / 48\right.$ ) for both distributions (Fig. 1a), allowing qualitatively different distributions to be compared. Values drawn from the beta distribution are limited to the range $[0<\alpha<1]$ and characterised by 2 parameters, $\mathrm{p}$ and $\mathrm{q}$ which combine to give the expected value $\left(\mu_{\beta}\right)$ and variance $\left(\sigma_{\beta}^{2}\right)$

$$
\begin{aligned}
\mu_{\beta} & =\frac{\mathrm{p}}{\mathrm{p}+\mathrm{q}} \\
\sigma_{\beta}^{2} & =\frac{\mathrm{p}+\mathrm{q}}{(\mathrm{p}+\mathrm{q})^{2}(\mathrm{p}+\mathrm{q}+1)}
\end{aligned}
$$

Under parameter combinations $\mathrm{p}=\mathrm{q}=1$, the beta distribution is equivalent to a uniform distribution over the limits $[0<\alpha<1]$. By choosing appropriate parameter values, a beta distribution can be generated where weak interactions dominate (Fig. 1a), as is thought to be the case in natural communities (McCann et al. 1998, Berlow 1999, McCann 2000). The expected value and variance or the uniform distribution can be found as

$$
\begin{aligned}
\mu_{u} & =\frac{a+b}{2} \\
\sigma_{u}^{2} & =\frac{(b-a)^{2}}{12}
\end{aligned}
$$

where $\mathrm{a}$ and $\mathrm{b}$ are the minimum and maximum values. By setting parameter a to 0 and solving for the remaining parameters, the values for the beta-distribution that correspond to the same expected value and variance $\left(\mu_{\beta}=\mu_{u}\right.$, $\left.\sigma_{\beta}^{2}=\sigma_{\mathrm{u}}^{2}\right)$ are

$$
\begin{aligned}
& \mathrm{p}=3-2 \mathrm{~b} \\
& \mathrm{q}=\frac{(2 \mathrm{~b}-3)(\mathrm{b}-2)}{\mathrm{b}}
\end{aligned}
$$

giving $\mathrm{p}=2, \mathrm{q}=6$ for $\mu=1 / 4$ and $\sigma^{2}=1 / 48$. This allows a direct test of whether it is these moments of the distribution that affect feasibility and stability in competitive communities.

\section{Community assembly}

Communities were assembled following a sequential algorithm using the two different distributions described above. Communities were initiated with 2 species, with $\alpha_{i j}(i \neq j)$ values drawn at random form the relevant distribution. All $S=2$ communities were feasible and locally stable (F-LS). A new species was added to this pair, with all $\alpha_{i j}$ values corresponding to the invading species again drawn at random from the relevant distribution, and the new community tested for feasibility and local stability. If the community was F-LS, the process of adding new species was repeated, until a community that was not F-LS was found. Simulations were then used to determine the outcome of invasion. The
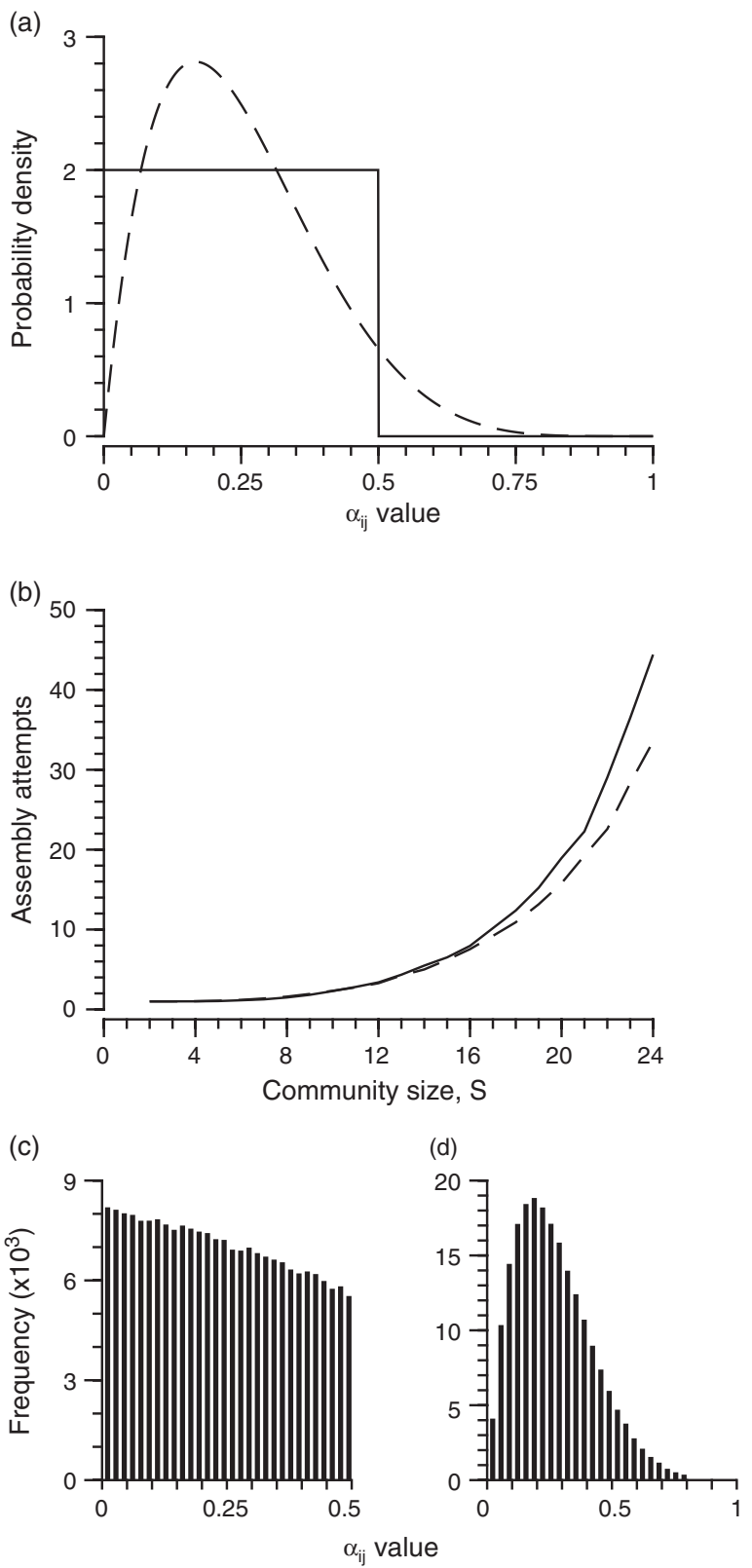

Figure 1. (a) The distribution of interaction strengths in a global species pool used to form communities. Two different distributions were examined, uniform (solid line) and beta (dashed line). Both distributions had the same expected value $(\mu=1 / 4)$ and variance $\left(\sigma^{2}=1 /{ }_{48}\right)$. (b) Number of extra invasion attempts required to assemble communities of different sizes from the distributions in panel (a). Examples of the realised distribution are given for interaction strengths pooled from 1000 replicates of 15 species communities, assembled from (c) uniform and (d) beta distributions.

new species was added to the last F-LS community at a low density $\left(\mathrm{N}_{\mathrm{i}, 1}=10^{-6}\right)$, the system was initiated with all other species at their previous equilibrium densities $\left(\mathbf{N}^{*}\right.$ evaluated for the previously F-LS, S-1 species community) and simulated over 10000 time-steps. After the simulation, the least abundant species was removed if its density declined constantly over the last 100 time steps of the simulation, and the remaining community tested again. If it was F-LS, a new species was added and the building process continued. 
If it was not F-LS, the community was simulated again for 10000 steps, being initiated at the densities found at the end of the previous simulation round, with the exception of the least abundant species, which had already been removed. This removal process continued until a F-LS community was found, when a new species was added and the process continued as above. The number of extra invasion attempts required to form each $S$ species community was recorded for both distributions.

The realised $\alpha_{i j}$ values were recorded for a sample of 1000 F-LS randomly and sequentially assembled communities for all $S$. These values were pooled and used to derive parameter values using maximum likelihood estimates (MLEs) for each $S$, for the relevant distribution. The pooled interaction values were compared to cumulative distributions from both the original global distribution and the distributions derived from the MLE parameter values using a Kolmogorov-Smirnov goodness of fit test. This tested whether the assembled distribution shape changed compared to the initial global distribution, and whether the same type of distribution could be used to adequately describe any change that occurred.

A random community assembly method was also investigated for both distribution types (Supplementary material Appendix 1).

\section{Species deletion and cascading extinctions}

To examine the effect of species deletion on communities formed using the different distributions, a sample of 1000 F-LS communities were investigated over the range $S=2$ to 24 . For each replicate, the relative abundance rank of each community member was evaluated. The least abundant (ranked 1), most abundant (ranked S), or a random species were each removed independently from the community, and the feasibility and local stability of the remaining community was evaluated. If the remaining community was not F-LS, it was simulated to assess which species were involved in extinction cascades. These communities were initiated with species at their S-species equilibrium densities, with the exception of the deleted species $\left(\mathrm{N}_{\mathrm{i}}=0\right)$. The $\mathrm{S}-1$ species community was simulated; the least abundant species after 500 time-steps was removed and its relative equilibrium abundance (in the S-1 species community) recorded. The new (S-2) species community was evaluated for F-LS, and simulations repeated until a F-LS species was found. The abundance ranks of species lost through extinction cascades were compared to their relative abundance in the full S-species community to determine how often species of different abundance were involved in extinction cascades. Equilibrium densities were re-evaluated following each extinction to determine the system attractors and evaluate how reorganisation following deletion was involved with species loss.

The interaction strengths of all communities was recorded and pooled into two groups (those that persisted or lost species) following the primary extinction for further analysis. All simulations and statistical analyses were carried out in MatLab 7.4.0 (R2007a).

\section{Results}

\section{Community assembly}

As community size increased, it became more difficult to assemble communities, and more so for communities with interaction strengths drawn from a uniform compared to a beta distribution (Fig. 1b). The distribution of interaction values did not differ from the global distribution in small communities ( $\mathrm{S} \leq 6$ for both the uniform and beta distributions), but did for larger communities (Fig. 1c-d, Table A1). While the MLEs for the beta distribution accurately described the interaction values, they did not in uniform communities when $S \geq 7$ (Table A1, Fig. 2a-b, A3, A4). The mean of the distribution of interaction values predicted by the MLEs was compared with the sample mean of the realised interaction values (Fig. 2c-d). As $S$ increased, the mean interaction strengths in the assembled communities declined. This was predicted by the MLEs for the beta distribution, but not for the uniform. The median and variance of the interaction values also declined with increasing $S$ (Fig. 2e-f). The MLEs performed poorly for describing communities drawn from the uniform distribution, because interaction strengths were skewed away from a uniform distribution to weaker interactions (Fig. 1c, A4): the sample data did not match the MLE's assumption of a uniform distribution.

These general patterns did not hold for randomly assembled communities, with no important differences between distribution types in community assembly (Fig. A1), although the assembled beta communities did have a lower median interaction strength than random uniform communities (Fig. A2). Random communities were more difficult to assemble than under the sequential algorithm.

\section{Species deletions and cascading extinctions}

The probability of extinction cascades following species removal increased with increasing community size with both global distributions (Fig. 3a). Removal of the least abundant species led to extremely few cascading extinctions. Deleting a random or the most abundant species respectively increased the probability of further species loss. Extinction cascades were more likely in uniform communities than beta (compare the solid and dashed lines in Fig. 3a). Those communities that were not F-LS were always unfeasible (at least one $\mathrm{N}_{i}^{*} \leq 0$ ) rather than being feasible and locally unstable. Extinction cascades were less likely to occur in sequentially than randomly assembled communities (Fig. A5).

Similar patterns arose in the mean number of species lost in extinction cascades (Fig. 3b). More species were lost when the most abundant species was removed than a random or the least abundant species and fewer species were lost when the community was drawn from a beta distribution than a uniform. Sequentially assembled communities also lost fewer species than randomly assembled (Fig. A5).

These results are hard to explain given the similarity in the sample mean interspecific interaction strengths of uniform and beta communities (Fig. 2c-d), but can be better understood through differences in the median (Fig. 2e) and variance (Fig. 2f) of pooled interaction strengths. Beta communities were more stable, lost fewer species and had lower median and variance in interaction strengths. 

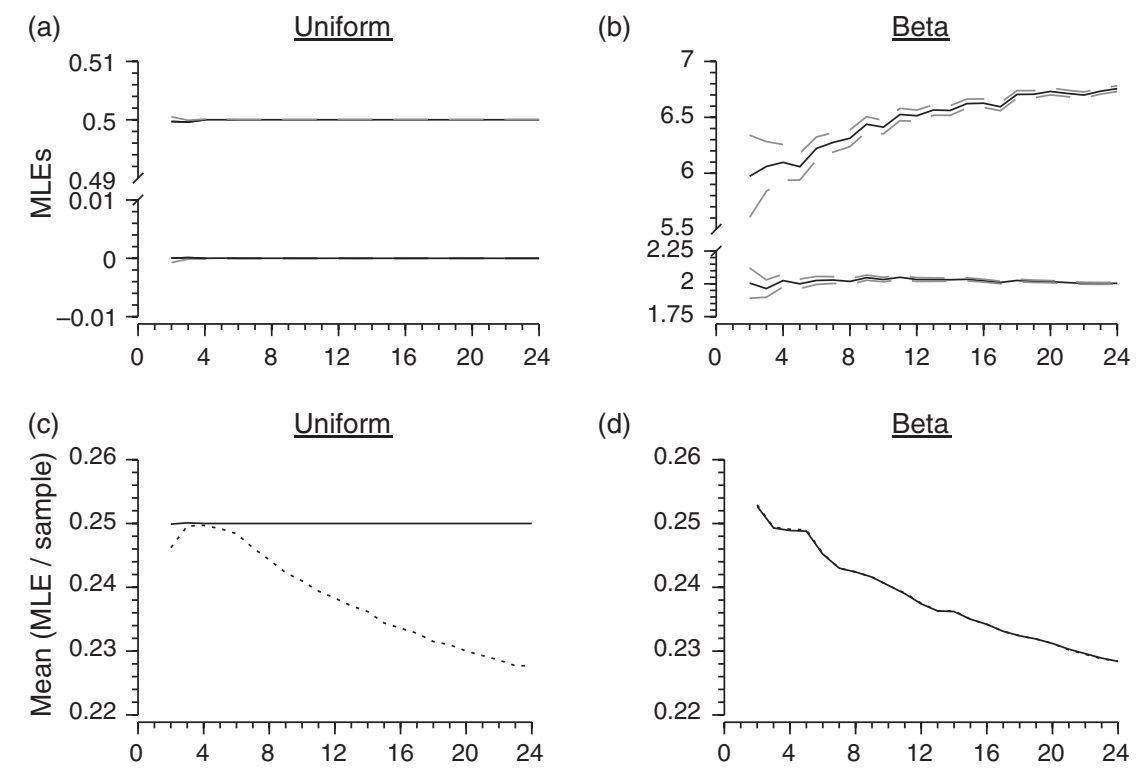

(d)
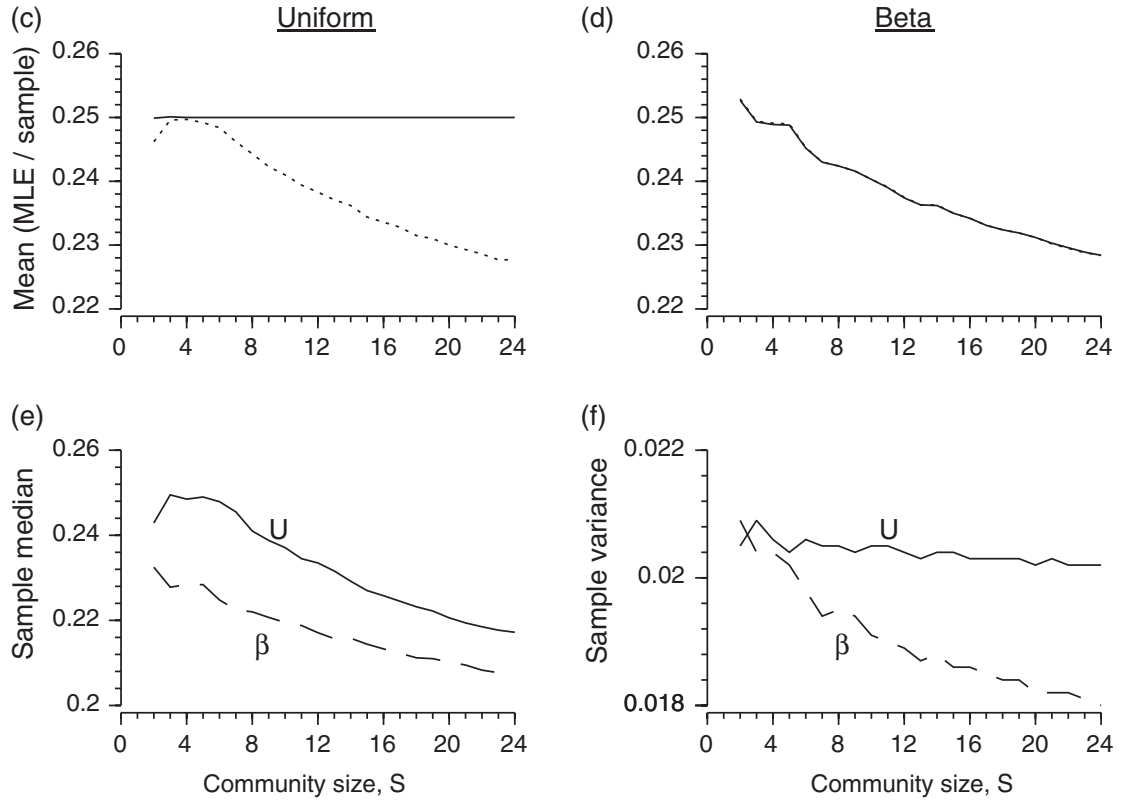

Figure 2. Properties of the interaction strengths $\left(\alpha_{\mathrm{ij}}\right)$ in sequentially assembled competitive communities with species interactions drawn from a uniform or beta distribution. (a, b) Maximum likelihood estimates ( $\pm 95 \% \mathrm{CI}$, dashed lines) are used to derive the mean interaction strength $(\mathrm{c}, \mathrm{d})$ for the interspecific interaction values from 1000 feasible and locally stable communities from uniform (a, c) and beta (b, d) global distributions. Dotted lines in (c, d) show the sample mean interaction strengths, which match those from the MLEs for the beta distributions but not the uniform. Panels (e and f) show the sample median and variance for all uniform (solid) and beta (dashed lines) distribution types.

The community that remained following a forced extinction event (S-1 species) was more likely to be stable than a randomly assembled (S-1) community of equivalent size. This result held regardless of the global distribution, abundance rank of species removed or assembly method (Fig. A6, Table A2).

\section{Which species are lost in extinction cascades and why?}

Results presented here are based on removal of the most abundant species, however there was no qualitative difference with results following the removal of a random species. The relative abundance rank of species involved in extinction cascades varied considerably, when based on rank in the original (pre-deletion) community, though less abundant species were more likely to be lost (Fig. 4a-b). When species deletion led to an extinction cascade, this was always associated with structural (unfeasible, $\mathrm{N}_{\mathrm{i}}^{*} \leq 0$ ) rather than dynamical instability (feasible but locally unstable, $\left|\lambda_{1}\right|>1$ ). The reorganization that occurred within the community following the primary removal of the most abundant species, as well as reorganization following subsequent extinction events, meant that those species that were involved with extinction cascades were nearly always the least abundant (rank 1; Fig. $4 \mathrm{c}-\mathrm{d})$ and had generally had negative equilibrium densities when the reorganised community was considered - less than $1 \%$ of species lost had $\mathrm{N}_{\mathrm{i}}^{*}>0$, regardless of community size, global distribution or assembly method. In the simulations, species tending to extinction with $\mathrm{N}_{\mathrm{i}}^{*} \leq 0$ declined asymptotically to 0 , but could not take densities $<0$.

Communities that suffered from cascading extinctions following removal of the most abundant species (unfeasible) had a higher sample mean and variance in interaction strengths than those that did not suffer further extinctions (F-LS; Fig. 5). Small, unfeasible post-removal (S-1) beta communities had a higher sample mean and variance than those from a uniform distribution, explaining the higher number of species lost from $S=4$ beta communities (Fig. 3b).

\section{Discussion}

When species are drawn from a global pool with interaction strengths described by different distributions that shared 
(a)

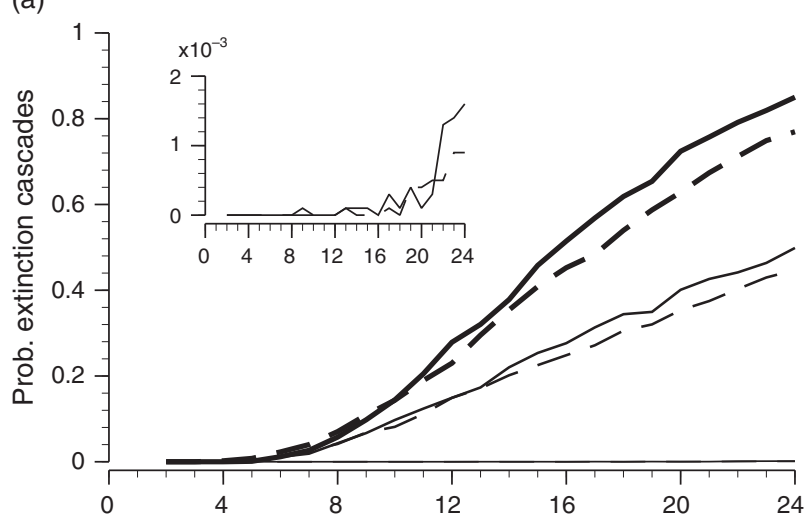

(b)

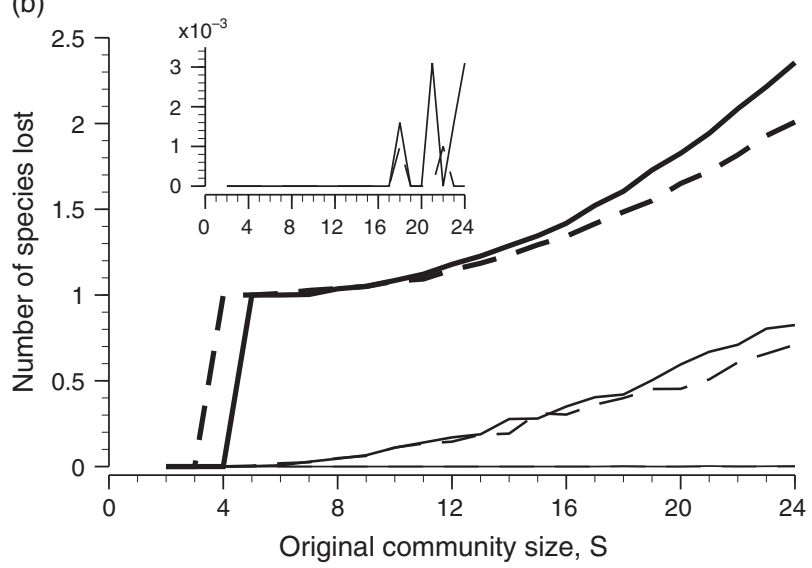

Figure 3. Community responses to forced extinction events. (a) The probability of extinction cascades and (b) the mean number of species lost, in $S$ species communities following the removal of the least abundant (thin lines), a random (intermediate) or the most abundant species (thick lines). Communities were formed from a uniform (solid lines) or beta (dashed lines) distribution. Results are based on 1000 F-LS communities for each scenario. Removing the most abundant species led to extinction cascades more often, with more species lost. Beta communities were more robust to species deletion and lost fewer species than those drawn from a uniform distribution. Inlays show the magnified results for least abundant species removal.

a common mean and variance (Fig. 1a), it is harder to sequentially assemble communities from a uniform global distribution than a beta distribution (Fig. 1b). Beta communities are more robust to species removals, with a lower probability of extinction cascades and fewer species lost in these cascades than uniform communities. These differences can be explained by relative differences in the median and variance of the actual interaction strengths between competitors in the assembled communities, which were both lower in beta than uniform communities (Fig. 2) and higher in communities that lost species in extinction cascades than those that did not (Fig. 5). The distribution of interaction strengths in communities drawn from a global uniform distribution was clearly skewed to weaker interactions, and a non-uniform distribution shape (Fig. 1c, A1). Interactions in assembled beta communities also differed from their original global distribution (Table A1).
Random assembly methods were also tested for both distributions (Supplementary material Appendix 1), with extinction results mirroring those for sequentially assembled communities with increasing community size, but little difference in assembly or post species removal characteristics between distribution types for randomly assembled communities (Fig. A5). Sequentially assembled communities were more robust to species removal than randomly assembled (Fig. A5).

There were clear differences in the probability of extinction cascades according to the abundance rank of the species removed (Fig. 3, Fowler 2005), which also reflects differences in the fragility of ecological networks to random or selective removal of nodes (Montoya et al. 2006). Extinctions occurred across the whole community (following removal of the most abundant species), but these results were based on species' abundance ranks in the original community (Fig. $4 \mathrm{a}-\mathrm{b})$. Species lost in extinction cascades were nearly always found to be the least abundant (Fig. $4 \mathrm{c}-\mathrm{d}$ ), when considered in the context of re-organised, post-extinction communities, generally having negative equilibrium densities (Morton et al. 1996). Communities that suffered extinction cascades had a higher mean and variance in interaction strength than those that did not lose further species, but this became harder to distinguish as community size increased (Fig. 5).

The fact that these secondary extinction cascades can occur in single trophic level communities where species compete over some limiting resource shows that facilitation through indirect interactions promotes coexistence. Invasion order is therefore a crucial component of community assembly (Morton et al. 1996, 1997). This provides insight into another route to secondary extinctions that does not involve removal or depletion of limiting resources, which may also play a role in trophically structured community models.

Identifying which species are likely to be involved in extinction cascades in complex, natural ecosystems is unlikely to be straightforward. Relying on species relative abundance, based on the original, pre-removal community highlights how variable the outcome can be (Fig. 4). Further species loss may occur before the reorganised community settles and preventative measures can take place to protect those (least abundant) species most at risk. Recent work has shown that 'trophically unique' species are involved in extinction cascades more often than by chance (Petchey et al. 2008). Calculation of trophic uniqueness requires highly resolved foodwebs with detailed information about interaction strengths, information that is generally hard to get, or unavailable from natural systems (Abrams 2001). Is species relative abundance rank easier to find than the trophic diversity in natural systems? This will be dependent on the complexities of the particular study system under consideration, e.g. microcosm (Fox and McGrady-Steed 2002) versus field (Paine 1966) food-web studies, but knowledge of abundance rank will clearly provide useful, easily accessible information about which species are dangerous to remove from communities, as well as what the knock on effects to the rest of the assemblage may be.

The presence of both weak (McCann et al. 1998, Berlow 1999) and strong (Jonsson et al. 2006) interactions has been highlighted in the past as being of importance in community structure and stability. The mean and variance of interaction values is also of importance in community assembly 

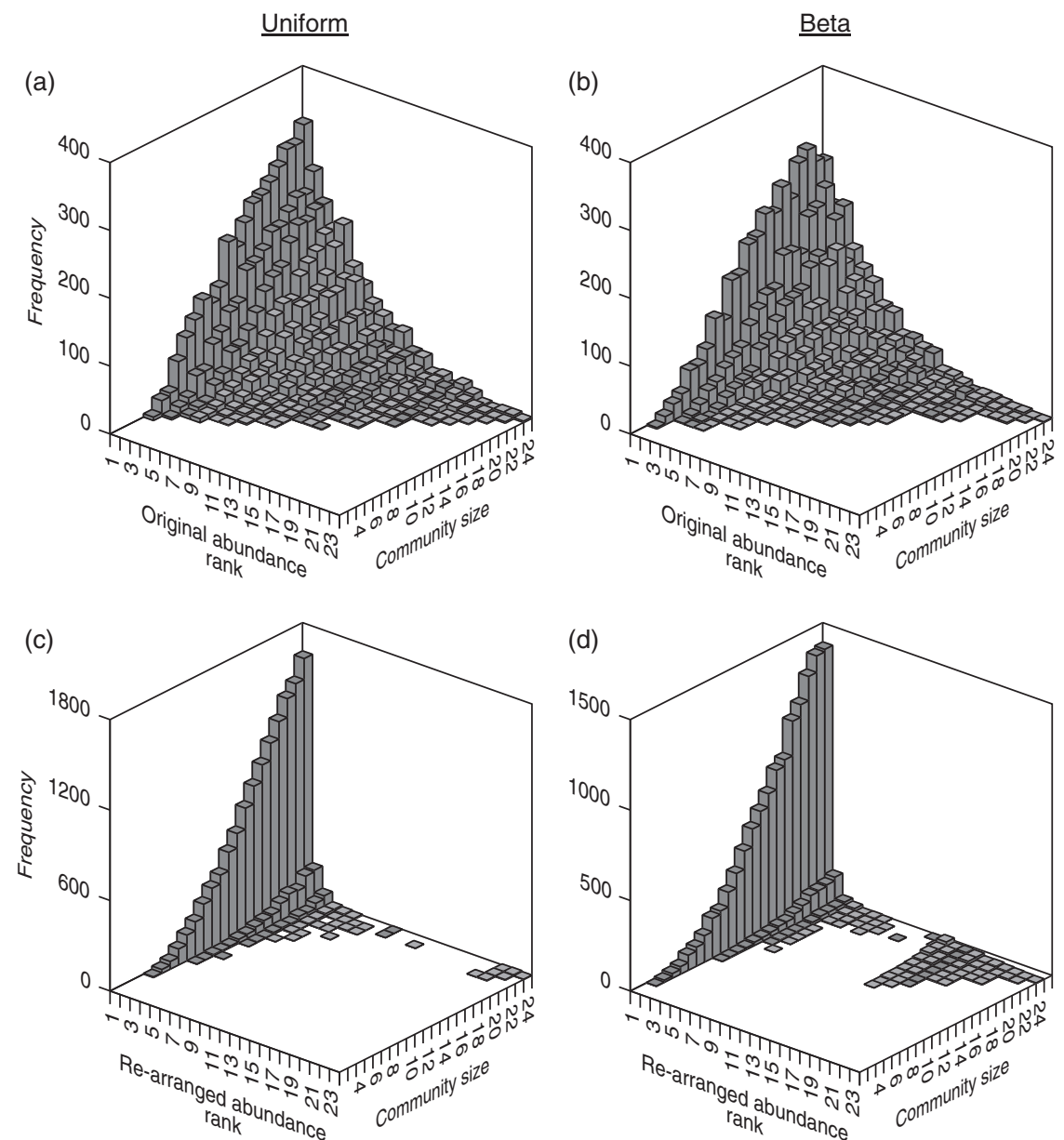

Figure 4. Which species are lost in extinction cascades? The frequency of extinction for each species are shown, characterised by their relative abundance ( 1 = least abundant, $\mathrm{S}=$ most abundant) in $(\mathrm{a}, \mathrm{b})$ the original community or $(\mathrm{c}, \mathrm{d})$ recalculated equilibrium densities after accounting for species loss, from 1000 F-LS communities following the removal of the most abundant species, when communities are sequentially assembled from a uniform $(\mathrm{a}, \mathrm{c})$, or beta $(\mathrm{b}, \mathrm{d})$ distribution. While the least abundant species in the original community are most commonly involved in extinction cascades, there are many instances when more abundant species are also lost ( $a, b)$. However, these more abundant species were generally found to have shifted down the abundance rank following community reorganisation (c, d).

and stability (reviewed by Berlow et al. 2004, see also Fowler et al. 2002, Jonsson et al. 2006, Fowler 2009). The beta distribution investigated here has the potential for stronger interaction strengths than a uniform distribution with the same mean and variance, as well as arguably a higher concentration of relatively weak interactions (Fig. 1a), therefore further details about the actual distribution may be required before generalities can be drawn. Emmerson and Yearsley (2004) investigated the influence of different interaction strength distributions on the assembly of simple four-species foodwebs, although they didn't maintain a constant variance between distribution types. They also noted skewed interaction distributions arising as an emergent feature of the assembled communities (Fig. 1c-d, A4), but in some cases, Emmerson and Yearsley (2004) showed interactions skewed towards stronger values, e.g. between basal prey and predators. Results presented here demonstrate that the distribution shape has a clear impact on community assembly and extinction properties; and that those community members that successfully coexist will have a skewed distribution of interaction strengths, even when drawn from a uniform distribution (Fig. 1c, A4).
Species deletion in model communities, as investigated here and elsewhere (Lundberg et al. 2000, Dunne et al. 2002, Fowler et al. 2002, Ebenman et al. 2004, Fowler 2005, Borrvall et al. 2006, Eklöf et al. 2006, Petchey et al. 2008), may represent an unrealistic situation for extrapolation to natural systems. Species may be more likely to experience a gradual change in their population size through factors such as harvesting or stocking of themselves or other species (Enberg et al. 2006), persecution, long-term climate change or the invasion of alien species. Studies that address these complex interactions represent a useful future direction for further research in model communities.

Deletion and reinvasion of deleted species from communities can change the feasibility and local stability of communities following such changes in species composition. In some cases, communities that experienced cascading extinctions demonstrate community closure, where the original species deleted cannot be reintroduced without causing further extinctions (Lundberg et al. 2000). This will not lead to further extinctions if none occurred following the original deletion (Fowler et al. 2002) and can be understood in terms of feasibility and local stability in the reduced communities. 

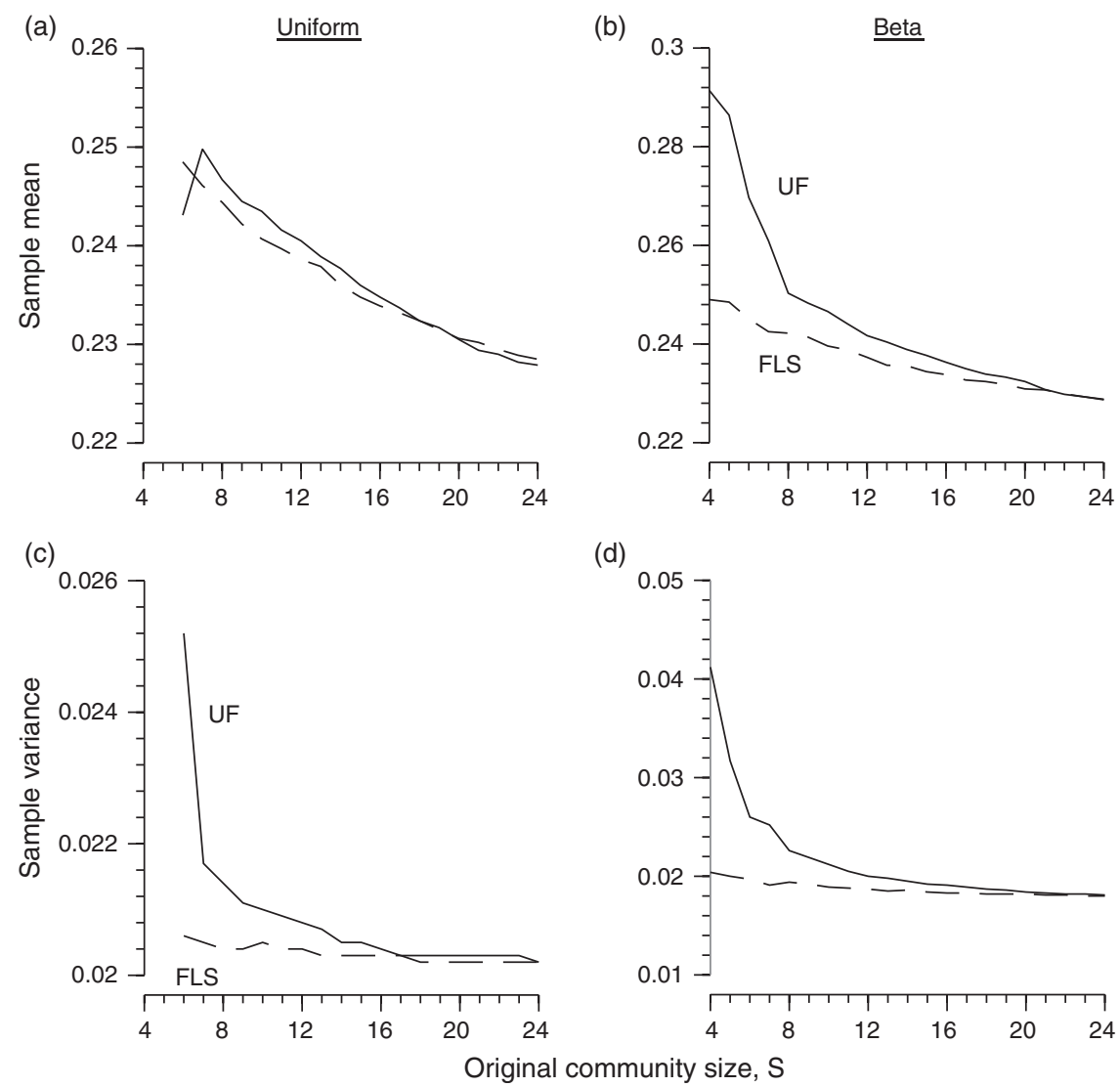

Figure 5. Communities that suffer from extinction cascades following initial species removal (unfeasible, solid lines) had a higher sample mean $(a, b)$ and variance $(c, d)$ in interaction strengths than those that were robust to species loss (feasible and locally stable, dashed lines). Values were sampled from 1000 sequentially assembled communities with interaction strengths drawn from (a, c) uniform or (b, d) beta distributions.

If the primary extinction from an $S$ species community does not lead to secondary extinctions, the S-1 community is F-LS and reintroduction will safely take us back to the original S species F-LS community. If secondary extinctions do occur after the primary extinction, the $S-1$ community is not F-LS. There is therefore a chance that the reintroduction of the species originally removed will lead to a community that is not F-LS due to loss of species in the extinction cascade. Analysis of sample communities used here also shows that removal of species with negative densities from unfeasible communities does not always guarantee that remaining community is F-LS (Morton et al. 1996). None of the post extinction communities examined here were feasible but locally unstable $\left(\left|\lambda_{i}\right|>1\right)$, i.e., extinction cascades were due to structural $\left(\mathrm{N}_{i}^{*} \leq 0\right)$ rather than dynamical instabilities.

Interest in the effects of species composition and assembly processes on community properties and ecosystem functioning remains high (Petchey et al. 2004 Thébault et al. 2007). While it will be interesting to compare the effects of different interaction strength distributions in more complex food-webs (Emmerson et al. 2004, Petchey et al. 2008) and across different spatial scales (Loreau et al. 2001), it is worthwhile examining and understanding these in a simpler competitive scenario before extending the analysis to further trophic levels (Berlow et al. 2004). Comparison of results generated under different assumptions will ultimately allow us to realise which of these features have important effects on the particular community and food-web processes we are interested in. Questions for further study are whether there are characteristic differences in abundance distributions generated from models using qualitatively different distributions of interaction strengths (e.g. uniform or beta), which can be used to help estimation and/or prediction in natural systems? Or, does the distribution of interactions strengths affect community responses to (and species loss caused by) environmental variation (Ruokolainen et al. 2007, 2008).

The model framework used here represents a simple, strategic approach to investigate the effect of different distribution shapes of interaction strengths on community assembly and persistence. The model does not include explicit resource dynamics, as trophically structured models generally do. All species are assumed to show positive growth at low densities and will persist in the absence of competitors $\left(r_{i}>0\right)$. Species specific carrying capacity, $\mathrm{K}_{\mathrm{i}}$, can be set by a limiting resource that is not directly affected by changes in species density over time, e.g. the availability of light or space, including territories (Paine 1966, MacNally 1983, Royama 1992), rather than some limiting nutrient/resource that is depleted and not replenished with faster dynamics than population growth. Alternatively, resource dynamics could occur on a considerably faster time-scale than the species of the focal community. 
While it is not meant to closely match any specific natural system, the single trophic level model investigated here could represent a guild of (basal/resource) species in a trophically structured system (Emmerson and Yearsley 2004, Thébault et al. 2007, Petchey et al. 2008). The introduction of trophic interactions has been shown to reduce competitive exclusion within a trophic layer, promoting coexistence in both empirical (Paine 1974) and theoretical work (Brose 2008). Real ecosystems are highly complex structures, yet even the most highly resolved natural foodwebs have only recorded a fraction of the links between component species. The importance of parasitic relationships has generally been underappreciated (Lafferty et al. 2006), although food-web resolution is improving (Ings et al. 2009). Further examination of the distribution of interaction strengths within and across trophic levels will provide a fruitful avenue for future empirical and modelling research.

Gurevitch et al. (1992) reviewed the evidence for and importance of competition in structuring communities from field experiments across a wide range of taxa, noting that the importance of competition appeared to vary across trophic levels. Other recent literature highlights the importance of interspecific competition in structuring communities of Anolis lizards (Roughgarden 1995), bryophyte mosses (Mulligan and Gignac 2002), coral reefs (Cornell and Karlson 2000, McCook et al. 2001), plant communities in savannah, semiarid and arid regions (Fowler 1986, Foster and Tilman 2003) and in phytophagous insects (Kaplan and Denno 2007), to name just a few examples.

Ground-beetle assemblages (Carabidae) provide a useful natural illustration of competition across a large number of species in a given habitat. Niemelä (1993) reviewed the evidence for interspecific competition leading to resource partitioning in extant Carabid communities, including comparisons between observed and random assemblages. Carabids could provide a useful system to test model predictions. In boreal regions, harsh winters provide a clear annual (discrete) temporal structure, with generalist granivorous Carabids competing over the new seed set in the spring, before reproducing later in the year with the next generation overwintering as eggs. In Finland, Carabids attain very high species richness in open habitats such as meadows, semi-natural grasslands and golf courses. For example over 70 Carabid species have been identified from five golf courses in Finland (range 28 to 45 species per golf course), containing guilds of herbivorous, omnivorous or predatory species, although there is overlap in resource use within and between guilds (Johan Kotze and Stephen Venn pers. com.).

Empirical removal experiments often generate data that are too short and noisy, or do not monitor responses across the whole community, to be able to determine the long-term outcome of species deletion (Fowler 1981, Tinkle 1982). However, Aksenova et al. (1998) excluded dominant plant species from alpine tundra plots over 13 years, and found strong negative affects on the least abundant species in the community - results which are in line with the model predictions presented here. Simulation based studies are considerably more preferable than the complete removal of species from the field to prevent undesirable, unforeseen consequences, but predictions for any particular system need to be based on a model that is tailored specifically for that system.
The distribution of interactions strengths has important effects on community assembly, persistence and responses to perturbations, therefore requires attention and further investigation. Consideration of only the mean and/or variance in interaction strengths may not give enough information to understand differences in community responses to perturbation.

Acknowledgements - Thanks to Sami Aikio, Andreas Lindén, Lasse Ruokolainen, Veijo Kaitala, Esa Ranta and Per Lundberg for discussions on community interactions and/or comments on the MS. Funding was provided by the NCoE EcoClim project.

\section{References}

Abrams, P. A. 2001. Describing and quantifying interspecific interactions: a commentary on recent approaches. - Oikos 94: 209-218.

Aksenova, A. A. et al. 1998. Plant interactions in alpine tundra: 13 years of experimental removal of dominant species. Ecoscience 5: 258-270.

Allesina, S. and Bodini, A. 2004. Who dominates whom in the ecosystem? Energy flow bottlenecks and cascading extinctions. - J. Theor. Biol. 230: 351-358.

Allesina, S. et al. 2006. Secondary extinctions in ecological networks: bottlenecks unveiled. - Ecol. Modell. 194: 150-161.

Berlow, E. L. 1999. Strong effects of weak interactions in ecological communities. - Nature 398: 330-334.

Berlow, E. L. et al. 2004. Interaction strengths in food webs: issues and opportunities. - J. Anim. Ecol. 73: 585-598.

Borrvall, C. and Ebenman, B. 2006. Early onset of secondary extinctions in ecological communities following the loss of top predators. - Ecol. Lett. 9: 435-442.

Borrvall, C. et al. 2000. Biodiversity lessens the risk of cascading extinction in model food webs. - Ecol. Lett. 3: 131-136.

Brose, U. 2008. Complex food webs prevent competitive exclusion among producer species. - Proc. R. Soc. Lond. B 275: 2507-2514.

Bruno, J. F. and O'Connor, M. I. 2005. Cascading effects of predator diversity and omnivory in a marine food web. - Ecol. Lett. 8: 1048-1056.

Cornell, H. V. and Karlson, R. H. 2000. Coral species richness: ecological versus biogeographical influences. - Coral Reefs 19: 37-49.

Dunne, J. A. et al. 2002. Network structure and biodiversity loss in food webs: robustness increases with connectance. - Ecol. Lett. 5: 558-567.

Ebenman, B. et al. 2004. Community viability analysis: the response of ecological communities to species loss. - Ecology 85: 2591-2600.

Eklöf, A. and Ebenman, B. 2006. Species loss and secondary extinctions in simple and complex model communities. - J. Anim. Ecol. 75: 239-246.

Emmerson, M. and Yearsley, J. M. 2004. Weak interactions, omnivory and emergent food-web properties. - Proc. R. Soc. Lond. B 271: 397-405.

Enberg, K. et al. 2006. The impacts of different management strategies and environmental forcing in ecological communities. - Proc. R. Soc. Lond. B 273: 2491-2499.

Foster, B. L. and Tilman, D. 2003. Seed limitation and the regulation of community structure in oak savanna grassland. - J. Ecol. 91: 999-1007.

Fowler, M. S. 2005. Predicting community persistence based on different methods of community ranking. - Ann. Zool. Fenn. 42: 533-543. 
Fowler, M. S. 2009. Increasing community size and connectance can increase stability in competitive communities. - J. Theor. Biol. 258: 179-188.

Fowler, M. S. and Lindström, J. 2002. Extinctions in simple and complex communities. - Oikos 99: 511-517.

Fowler, N. 1981. Competition and coexistence in a North Carolina grassland. II. The effects of the experimental removal of species. - J. Ecol. 69: 843-854.

Fowler, N. 1986. The role of competition in plant-communities in arid and semiarid regions. - Annu. Rev. Ecol. Syst. 17: 89-110.

Fox, J. W. and McGrady-Steed, J. 2002. Stability and complexity in microcosm communities. - J. Anim. Ecol. 71: 749-756.

Gurevitch, J. et al. 1992. A meta-analysis of competition in field experiments. - Am. Nat. 140: 539-572.

Ings, T. C. et al. 2009. Ecological networks - beyond food webs. - J. Anim. Ecol. 78: 253-269.

Ives, A. R. and Cardinale, B. J. 2004. Food-web interactions govern the resistance of communities after non-random extinctions. Nature 429: 174-177.

Ives, A. R. et al. 2003. Estimating community stability and ecological interactions from time-series data. - Ecol. Monogr. 73: 301-330.

Jonsson, T. et al. 2006. Food web structure affects the extinction risk of species in ecological communities. - Ecol. Modell. 199: 93-106.

Kaplan, I. and Denno, R. F. 2007. Interspecific interactions in phytophagous insects revisited: a quantitative assessment of competition theory. - Ecol. Lett. 10: 977-994.

Lafferty, K. D. et al. 2006. Parasites dominate food web links. Proc. Natl Acad. Sci. USA 103: 11211-11216.

Loreau, M. et al. 2001. Biodiversity and ecosystem functioning: current knowledge and future challenges. - Science 294: 804-808.

Lundberg, P. et al. 2000. Species loss leads to community closure. - Ecol. Lett. 3: 465-468.

MacNally, R. C. 1983. On assessing the significance of interspecific competition to guild structure. - Ecology 64: 1646-1652.

May, R. M. 1973. Stability and complexity in model ecosystems. Princeton Univ. Press.

McCann, K. 2000. The diversity-stability debate. - Nature 405: 228-233.

McCann, K. et al. 1998. Weak trophic interactions and the balance of nature. - Nature 395: 794-798.

McCook, L. J. et al. 2001. Competition between corals and algae on coral reefs: a review of evidence and mechanisms. - Coral Reefs 19: 400-417.

Montoya, J. M. et al. 2006. Ecological networks and their fragility. - Nature 442: 259-264.

Supplementary material (available online as Appendix O17817 at <www.oikos.ekol.lu.se/appendix >). Appendix 1.
Morton, R. D. and Law, R. 1997. Regional species pools and the assembly of local ecological communities. - J. Theor. Biol. 187: 321-331.

Morton, R. D. et al. 1996. On models for assembling ecological communities. - Oikos 75: 493-499.

Mulligan, R. C. and Gignac, L. D. 2002. Bryophyte community structure in a boreal poor fen. II. Interspecific competition among five mosses. - Can. J. Bot. Rev. Can. Bot. 80: 330-339.

Niemelä, J. 1993. Interspecific competition in ground-beetle assemblages (Carabidae): what have we learned? - Oikos 66: 325-335.

Paine, R. T. 1966. Food web complexity and species diversity. Am. Nat. 100: 65-75.

Paine, R. T. 1974. Intertidal community structure. - Oecologia 15: 93-120.

Paine, R. T. and Trimble, A. C. 2004. Abrupt community change on a rocky shore - biological mechanisms contributing to the potential formation of an alternative stable state. - Ecol. Lett. 7: 441-445.

Petchey, O. L. et al. 2004. Species loss and the structure and functioning of multitrophic aquatic systems. - Oikos 104: 467-478.

Petchey, O. et al. 2008. Trophically unique species are vulnerable to cascading edxtinction. - Am. Nat. 171: 568-579.

Pimm, S. L. 1980. Food web design and the effect of species deletion. - Oikos 35: 139-149.

Quince, C. et al. 2005. Deleting species from model food webs. Oikos 110: 283-296.

Roughgarden, J. 1995. Anolis lizards of the Carribean. - Oxford Univ. Press.

Royama, T. 1992. Analytical population dynamics. - Chapman and Hall.

Ruokolainen, L. and Fowler, M. S. 2008. Community extinction patterns in coloured environments. - Proc. R. Soc. Lond. B 275: $1775-1783$.

Ruokolainen, L. et al. 2007. Extinctions in competitive communities forced by coloured environmental variation. - Oikos 116: 439-448.

Thébault, E. et al. 2007. Cascading extinctions and ecosystem functioning: contrasting effects of diversity depending on food web structure. - Oikos 116: 163-173.

Tinkle, D. W. 1982. Results of experimental density manipulation in an Arizona lizard community. - Ecology 63: 57-65.

Turrelli, M. 1978. A reexamination of stability in randomly varying versus determinstic environments with comments on the stochastic theory of limiting simlarity. - Theor. Popul. Biol. 13: 244-267. 


\section{Appendix 1}

\section{Methods}

\section{Randomly assembled communities}

I started by examining the probabilities of feasibility and local stability in randomly assembled competitive communities, with interaction strengths ( $\alpha$-values) drawn from uniform or beta distributions. Communities were assembled over a range of community sizes $(S=2$ to 24$)$, with $\alpha$-values drawn at random from the different distributions (mean $1 / 4$, variance $1 / 48$ ). Feasibility and local stability were assessed for each S-species community, and the probability of being either unfeasible (UF), feasible and locally stable (F-LS) or feasible and locally unstable (F-US) was calculated from 10000 replicates for each S and distribution form. 1000 sample F-LS communities were selected for further analysis, as outlined in the Methods section in the main text, to investigate the distribution of interaction strengths before and after primary deletion, as well as community responses to species deletion.

\section{Supplementary results}

\section{Randomly assembled communities}

Communities formed by drawing the interaction strengths at random from either beta- or uniform distributions with the same expected value $(\mu=1 / 4)$, and variance $\left(\sigma^{2}=1 / 48\right)$ were assembled to determine the effect of the global distribution of interaction strengths on assembly processes (Fig. A1). While there was a statistical difference in the probability of being F-LS between communities whose interaction strengths were drawn from either a beta- or uniform distribution [logistic regression results: uniform intercept $=5.259( \pm 0.024 \mathrm{SE})$, beta intercept $=4.817( \pm 0.022)$; uniform coefficient $=-0.523( \pm 0.002)$, beta coefficient $=-0.495$ $( \pm 0.002)]$, this was unlikely to be biologically important.

It was not straightforward to compare random or sequential assembly methods, however, it could be argued that it was easier to assemble large $\mathrm{S}$-species communities sequentially than randomly. For example, $\sim 1$ in 143 randomly assembled 20 species communities were F-LS (probability $=0.007$ for both uniform and beta distributions, Fig. A1), while sequential communities required on average $\sim 19$ (uniform) or 16 (beta) repeated invasion attempts to find a F-LS 20 species community.

The distribution of interaction values in randomly assembled communities differed from the initial uniform global distribution in medium and large communities $(S \geq 9$ in uniform and $S$ $\geq 7$ from beta distributions; Table A1, Fig. A2, A3). MLE's were not representative of the pooled data from randomly assembled uniform communities when $S \geq 9$ (due to an increase in intermediate, rather than weak, competition values), but differed only for three very large random beta communities $(S=19,21$ and 23). Small F-LS communities were easy to form, therefore matched the global distribution. The fact that interaction values differed to the original global distribution in larger communities shows that some selection (for species with intermediate interactions) occurred in the random assembly process (Fig. A3). Communities randomly assembled from a beta distribution had a lower sample median and variance than those from a uniform distribution, though neither declined much with increasing community size (Fig. A2e-f).

There was little difference in the responses of uniform and beta communities to primary species deletion (Fig. A5), either in the probability of extinction cascades or the number of species lost. As in sequentially assembled communities, increasing the abundance rank of the deleted species lead to an increase in both the probability of extinction cascades and the number of species lost. Randomly assembled communities were less robust and lost more species than sequentially assembled communities.

Another interesting question is whether a randomly assembled $\mathrm{S}$-species community (e.g. $\mathrm{S}=4$ species) is more likely to be feasible and locally stable than a $(S-1$, e.g. $S=5)$ community that has gone through primary species deletion. Figure A6 clearly shows that $\mathrm{S}-1$ communities are more likely to be stable than randomly assembled communities of the same size (Table A2). Again, beta communities are more stable than uniform, and sequentially assembled communities are more stable than randomly assembled. 


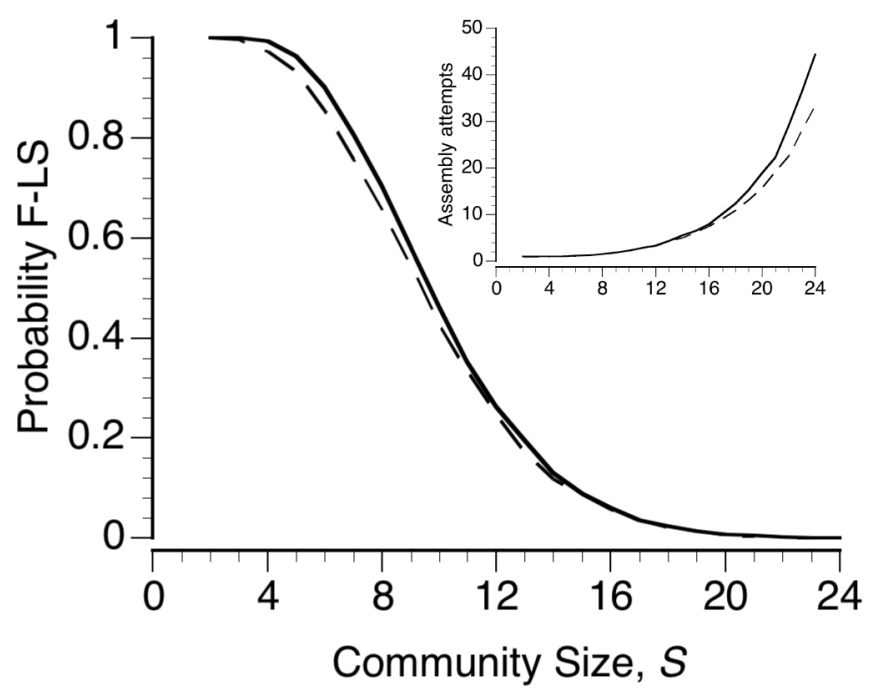

Figure A1. Increasing community size reduced the probability that randomly assembled communities would be feasible and locally stable (F-LS), when $\alpha_{i j}$ values were drawn from uniform (solid line) or beta (dashed line) global distributions. Inlay shows the number of invasion attempts required for sequentially assembled communities for comparison (Fig. 1b, main text). 
(a)

Uniform

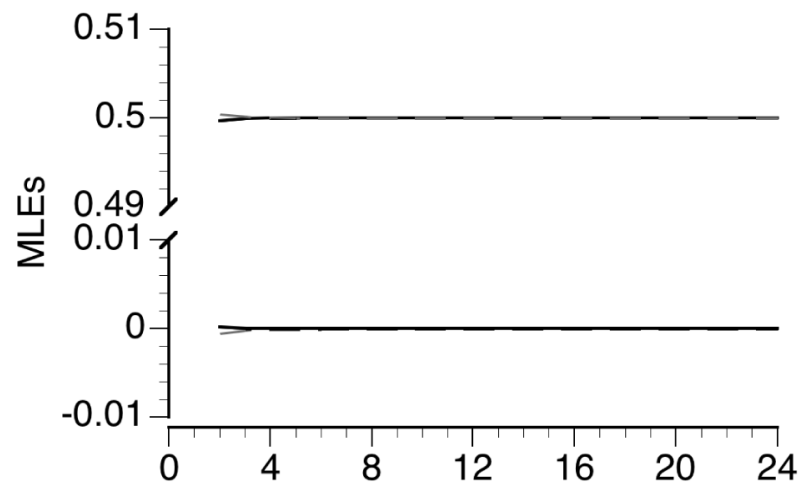

(c)

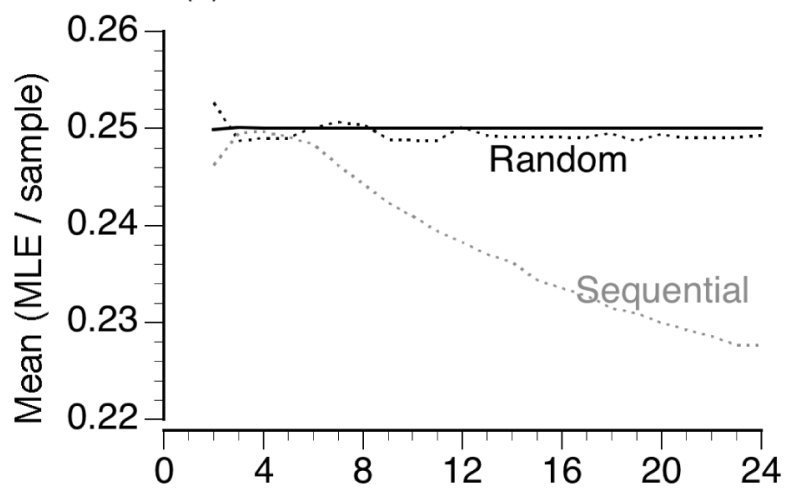

(e)

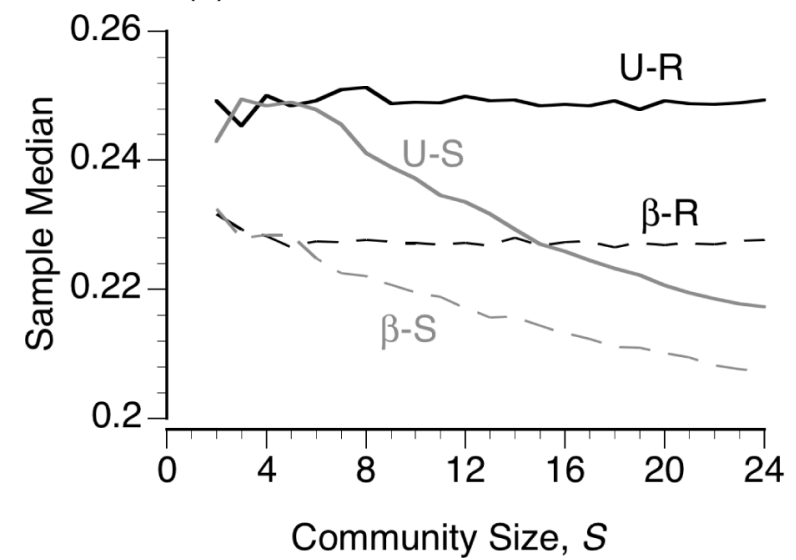

(b)

Beta

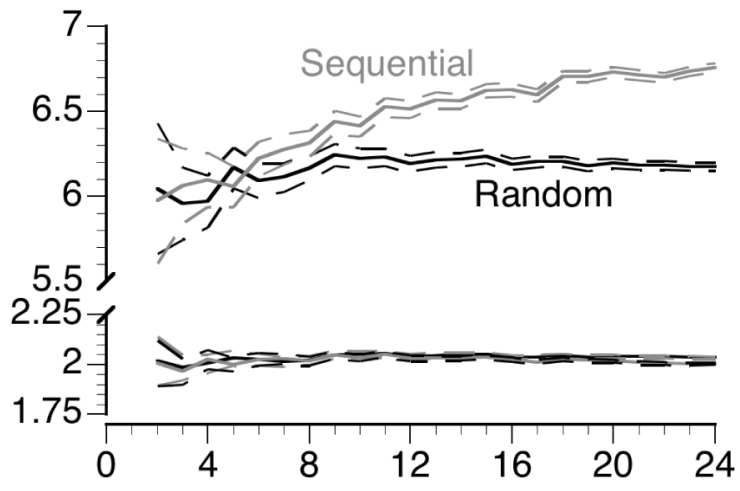

(d)

Beta

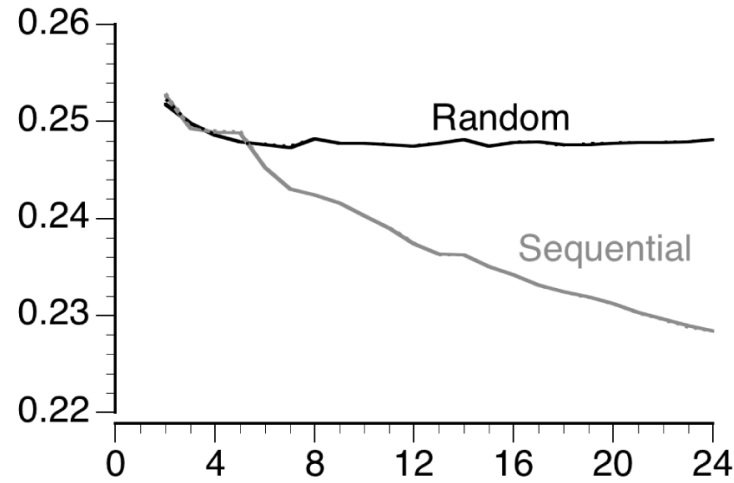

(f)

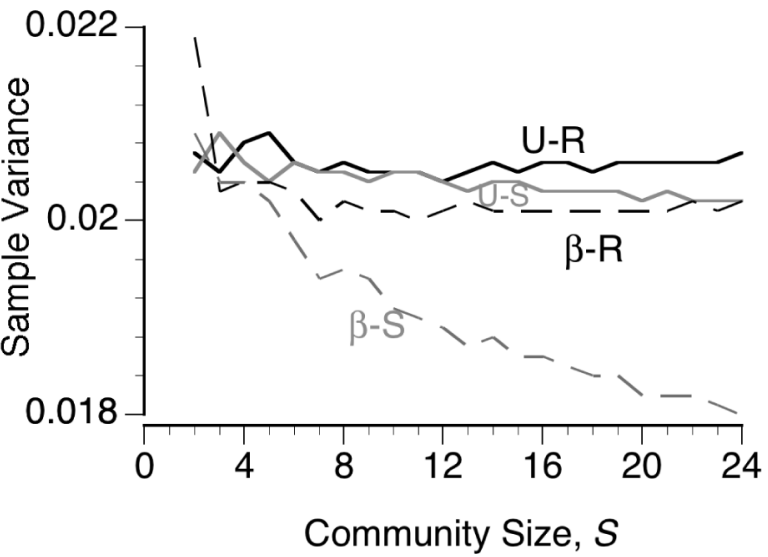

Figure A2. Properties of the interaction strengths $\left(\alpha_{i j}\right)$ in randomly assembled competitive communities (black lines) with species interactions drawn from a uniform or beta distribution. (a, b) Maximum likelihood estimates $( \pm 95 \%$ confidence intervals, dashed lines) are used to derive the mean interaction strength $(c, d)$ for the interspecific interaction values from 1000 F-LS communities from uniform $(a, c)$ and beta (b, d) global distributions. Dotted lines in (c, d) show the sample mean interaction strengths, which match those from the MLEs for the beta distributions but not the uniform. Panels (e and f) show the sample median and variance for all uniform (solid) and beta (dashed lines) distribution types. Grey lines show the same results from sequentially assembled communities for comparison (Fig. 2, main text). 


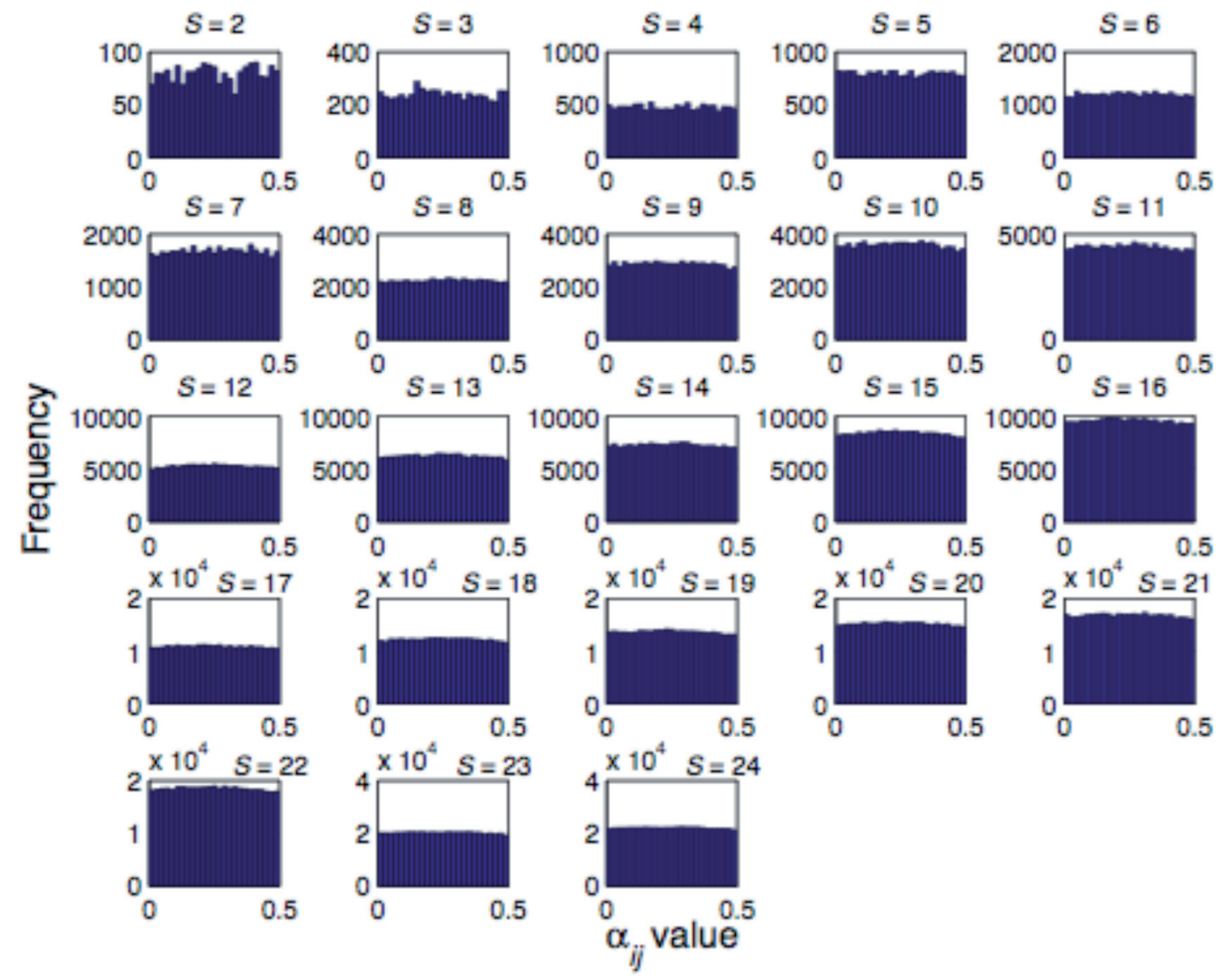

Figure A3. Pooled interaction strengths from 1000 randomly assembled communities with $\alpha_{\mathrm{ij}}$ values drawn from a uniform distribution. When $S \geq 9$, the distributions differed from both the initial global distribution (uniform distribution with limits $[0,0.5]$ ) and from a distribution using parameters derived by MLEs from the above data. This shows that the MLEs do not represent the data well in medium and large communities, due to an increase in intermediate interaction strengths $\left(\alpha_{\mathrm{ij}} \approx 0.25\right)$. 

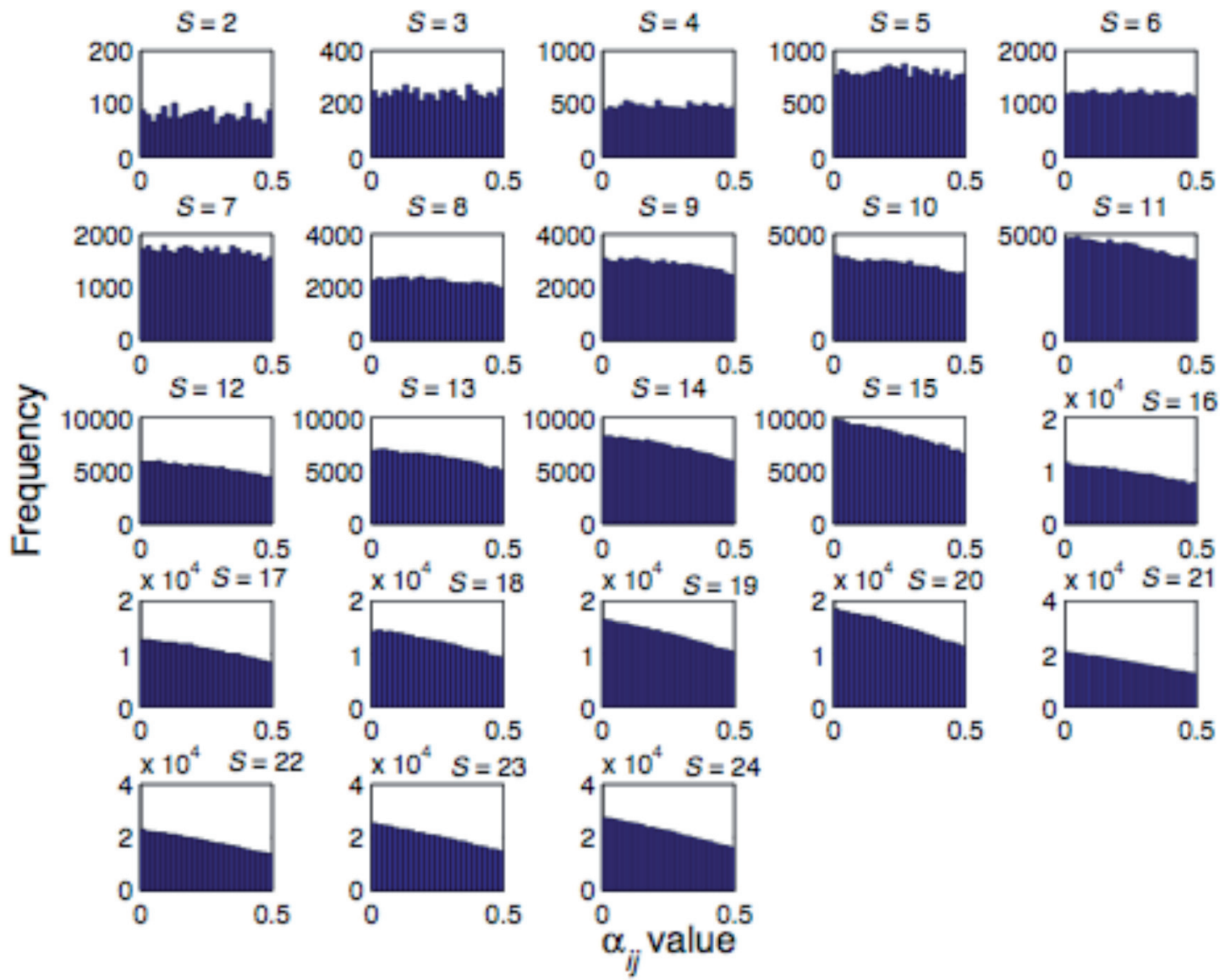

Figure A4. Pooled interaction strengths from 1000 sequentially assembled communities with $\alpha_{\mathrm{ij}}$ values drawn from a uniform distribution. When $S \geq 9$, the distributions differed from both the initial global distribution (uniform distribution with limits [0, 0.5]) and from a distribution using parameters derived by MLEs from the above data. This shows that the MLEs do not represent the data well in medium and large communities, due to an increase in weak interaction strengths. 
(a)

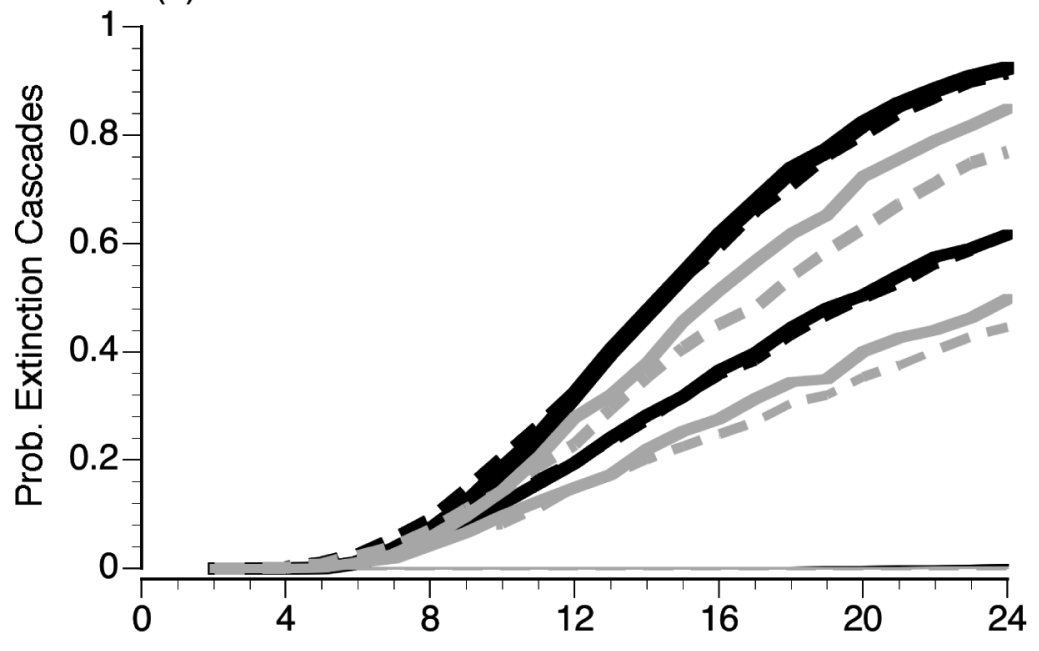

Dist - A. Meth - Rank Remvd

(b)

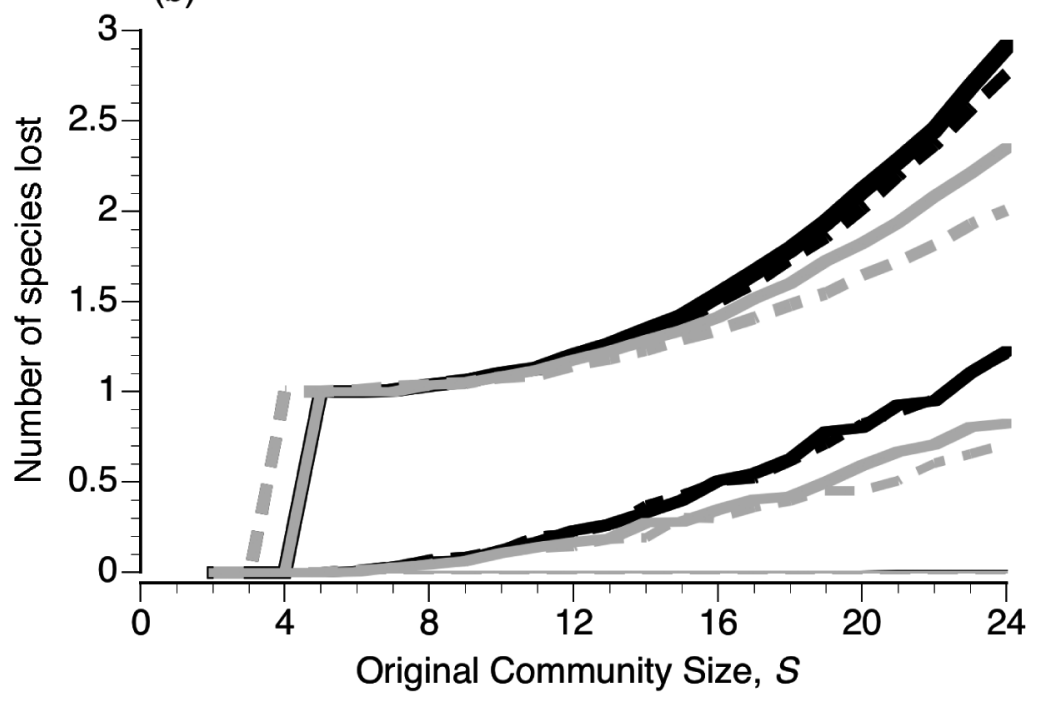

- - beta rand $\mathrm{L}$

— unif rand $\mathrm{L}$

- - beta rand $\mathrm{R}$

unif rand $R$

- - beta rand $\mathrm{M}$

unif rand $M$

- - beta seq L

— unif seq L

- - beta seq R

unif seq R

- - beta seq $\mathrm{M}$

unif seq M

Figure A5. Randomly assembled community responses to forced extinction events (black lines). (a) The probability of extinction cascades and (b) the mean number of species lost, in $S$ species communities following the removal of the least abundant (thin lines), a random (intermediate) or the most abundant species (thick lines). Communities were formed from a uniform (solid lines) or beta (dashed lines) distribution. Results are based on 1000 F-LS communities for each scenario. Removing the most abundant species led to extinction cascades more often, with more species lost. There was little difference between uniform and beta communitied. Grey lines show the equivalent results for sequentially assembled communities, which were more robust to species deletion. Legend shows the distribution type (beta, uniform), Assembly method (random, sequential) and rank abundance of the removed species (least, random, most). 


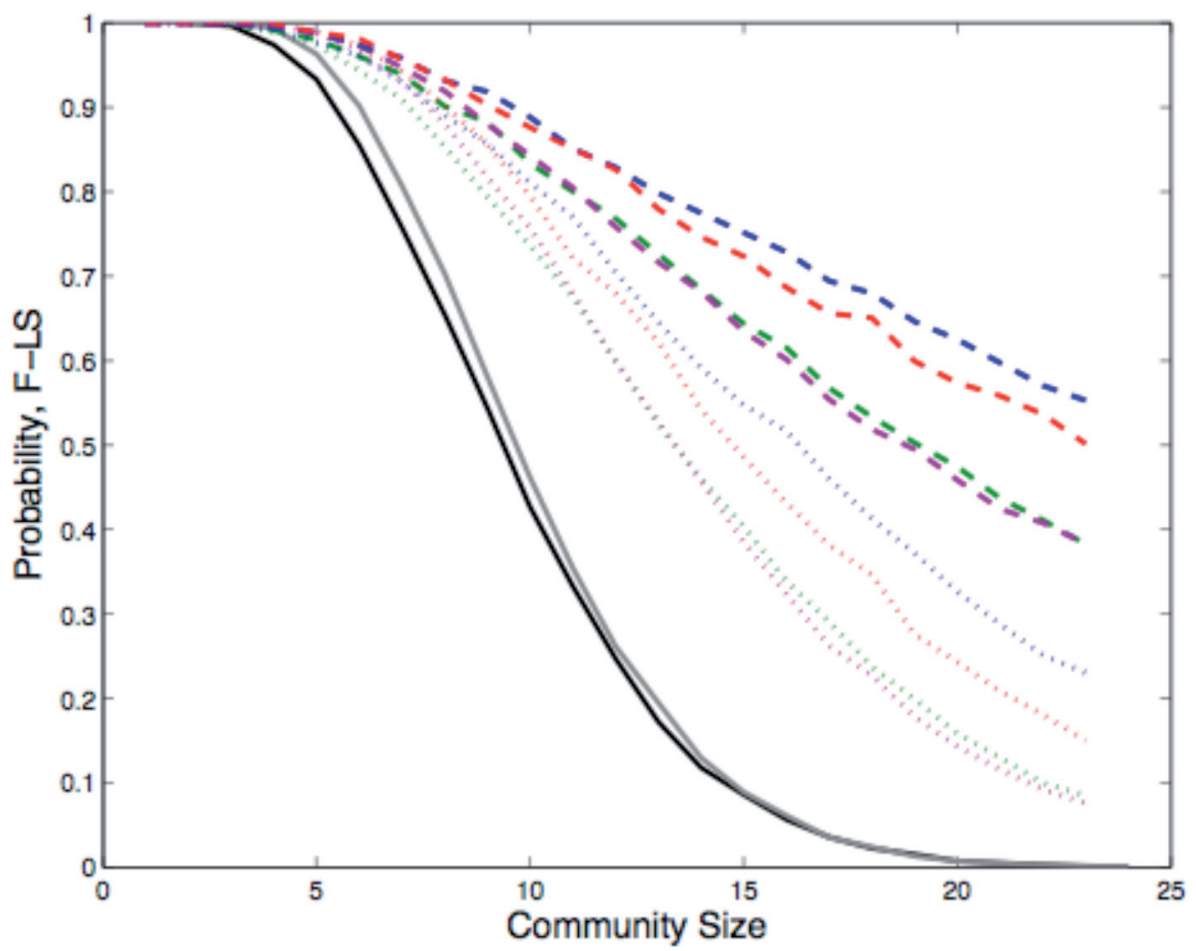

Figure A6. The probability of feasibility and local stability is lower in randomly assembled $S$ species communities (black line $=$ uniform distribution, grey line = beta distribution) than communities that contained S species following a primary extinction event. This holds regardless of which species was removed (most abundant $=$ dotted lines, random $=$ dashed lines), distribution type or the assembly process (blue $=$ beta sequential; red $=$ uniform sequential, green $=$ beta random, magenta $=$ uniform random). 


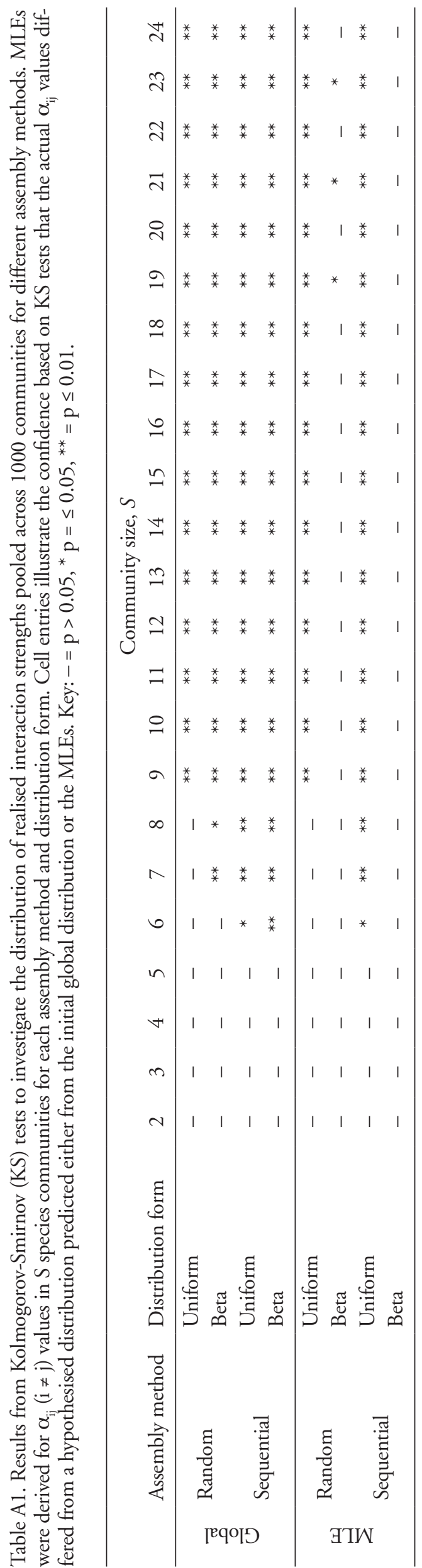


Table A2. Multiple logistic regression confirms that there is a significant difference between the least stable reduced communities (uniform random) and randomly assembled communities of the same size. The regression table shows results for the full model and partial statistics from a test of redundancy for each of the following explanatory variables: (1) intercept, (2) effect of community size on the probability of F-LS in randomly assembled communities from a uniform distribution, (3) effect of community size on the probability of F-LS in randomly assembled communities from a uniform distribution after the most abundant species had been removed, (4) difference in intercept between the two treatments.

\begin{tabular}{lcccccc}
\hline & Coefficient $( \pm$ SE) & Deviance (full) & Deviance (null) & $\mathrm{G}^{2}$ & $\mathrm{DF}$ & $\mathrm{p}$-value \\
\hline Full Model & & 508.58 & 33365 & 32856.45 & 3 & $<0.001$ \\
$(1)$ & $4.8022( \pm 0.0688)$ & & 10978 & 10470 & 1 & $<0.001$ \\
$(2)$ & $-0.4946( \pm 0.0066)$ & & 17660 & 17152 & 1 & $<0.001$ \\
$(3)$ & $-0.3369( \pm 0.0042)$ & & 13870 & 13361 & 1 & $<0.001$ \\
$(4)$ & $0.2472( \pm 0.0939)$ & & 515.49 & 6.9086 & 1 & 0.009 \\
\hline
\end{tabular}

